\title{
GSM Based Smart Security System Using Arduino
}

\author{
Alumona T.L ${ }^{1}$, Oranugo C.O ${ }^{2}$, Eze C.E ${ }^{3}$, Onyeyili.T.I ${ }^{4}$ \\ Department of Electronic and Computer Engineering, Nnamdi Azikiwe University, Awka ${ }^{1,2,3,4}$
}

\begin{abstract}
The need for security cannot be overemphasized in today's modern world, where residential and industrial fire outbreaks, gas leakages, over-current destructions, theft, suffocation from gas accumulations etc. are increasing alarmingly and becomes a major source of threat and environmental hazards. Current security systems in developing countries (if and when available) treat security as only intrusion based, and are of high operational and setup cost and thus the need for a smart and more integrated security system. The GSM based smart security system using Arduino presented employs the Passive Infrared (PIR), MQ2, MQ7 and the DHT 11 sensors in monitoring intrusion, combustible gases, carbon monoxide and temperature threats respectively and also incorporates remote monitoring and control capability. The concept of remote monitoring and control is implemented via the use of SMS and mobile phone calls respectively made possible by utilizing the SIM900 GSM module interface. The two operational modes - internal and external mode reduces the overall operational cost of the system and thus Remote monitoring (SMS), is used only when the owner is actually away, while audio and visual forms of alarms are utilized if he is close by the property. The entire system is controlled by the powerful Arduino Uno. The system resulted in a cost saving, smart, portable and a more efficient way of implementing security alarm systems.
\end{abstract}

Keywords: Arduino, remote monitoring, premises alarm security systems, GSM

\section{INTRODUCTION}

Security entails the condition of not being threatened, especially; physically, psychologically or emotionally [1]. A security system is a system designed to detect intrusion or unauthorized entry into a building or an area [2]. They are used in residential, commercial, military, and industrial properties for protection against burglary (theft) or property damage, as well as personal protection against intruders. Some security systems serve a single purpose of burglary protection. Combination systems provide protection for a number of threats and not just a single threat [2]. Conventional security systems tend to treat security as only burglar/intrusion inclined and, in the process, ignoring other potential threats. In today's society, threats ranging from; over-current destruction, suffocation arising from accumulation of carbon monoxide, fire (which can result from leakage and accumulation of combustible gases), leakage of dangerous gases etc., are all rampant, these threats also need to be monitored. The 'modern and smart' security systems which do in fact cover other potential threats and do function smartly by incorporating other forms of alarms notably phone-based alarms for remote monitoring, costs a fortune to set up, operate and maintain, owing to the nature of software design, type of materials used etc.

This design signifies a major improvement in terms of using cheaper materials, easier to maintain components and cheaper operating cost than other systems of the same kind, as notifications via text messages and calls are only made when owner is actually away. The GSM based smart security system using Arduino can be adopted at home, offices, institutions (educational, military, commercial, industrial, etc.). Much more importantly, considering the building arrangements in Nigeria, it is better suited to this terrain. It consists of various types of sensors used for the task of monitoring. In this work, the threats being monitored are those of intrusion (burglary), fire and dangerous gases. The system is fully controlled by the 'powerful' Arduino Uno board. The Arduino Uno board, continuously monitors all sensors, and if any security threat is detected from these sensors, then a combination of three alarms are triggered, namely; visual, audio and/or phone-based alarm. The system offers a 'safe' security system, in that only the user can activate or deactivate the two operational modes, through a phone call made only from his phone to the system, unlike other systems. This capability means that the security system is in itself minimally prone to attack.

\section{THE ARDUINO PLATFORM}

Arduino is an open source, computer hardware and software company, project, and user community that designs and manufactures single-board microcontrollers and microcontroller kits for building digital devices and interactive objects that can sense and control objects in the physical world [3]. It is a flexible programmable hardware platform designed for artists, designers, tinkerers, and the makers of things [4]. The Arduino project has grown exponentially since its introduction in 2005 [5]. Arduino is open-source electronics prototyping platform based on flexible, easy-to-use 


\section{IJARCCE}

hardware and software. In simple Terms, the Arduino is a tiny computer system that can be programmed with your instructions to interact with various forms of input and output [5]. The general impression however, is that the Arduino is a microcontroller, this is not the case as can be seen from the view of the Arduino official website. However, central to the Arduino platform is the microcontroller, which can be either one of ATmega8, ATmega168, ATmega328, ATmega1280, or ATmega2560 with varying amounts of flash memory, pins, and features. The Arduino is now preferred to the conventional microcontroller because of the following reasons;

- $\quad$ Ease of programming - owing to presence of many libraries of codes and no requirement for a programmer device to burn in codes to the device.

- $\quad$ Ease of hardware construction - owing to presence of special hardware called "shields". Shields are Arduinocompatible boards on a printed circuit expansion board, which plug into the normally supplied Arduino pin headers [2]. Shields can provide motor controls for 3D printing and other applications, Global Positioning System (GPS), Ethernet, liquid crystal display (LCD), and GSM shields etc. as such the designer need not be bothered about complexities of circuit design but more on functionality of software.

- Open source - it is actually cheaper to develop in Arduino than most microcontrollers. This is as because the Arduino IDE is free, other pre-built libraries can also be gotten free of charge, unlike say the PIC or AVR compilers and IDEs whose costs can reach as high as \$50 [2]. Amongst other advantages

\subsection{Motion Sensors}

The motion sensors are a family of sensors that detects movement in any covered area. The motion might be made by an intruder, or made in a protected area (example the control room of an organization). The basic types of motion sensors include; infrared and ultrasonic motion sensors, which are the most popular, there is also the microwave type. These are all subdivided into active and passive types. The active types emit energy and monitors interruption of these, while the passive types wait passively for a change in the normal energy levels of the parameter being monitored.

1. Active Infrared Sensor: Active infrared detectors emit a constant light beam, which connect to a light detector. If the infrared beam is interrupted, the detector sounds the alarm [6].

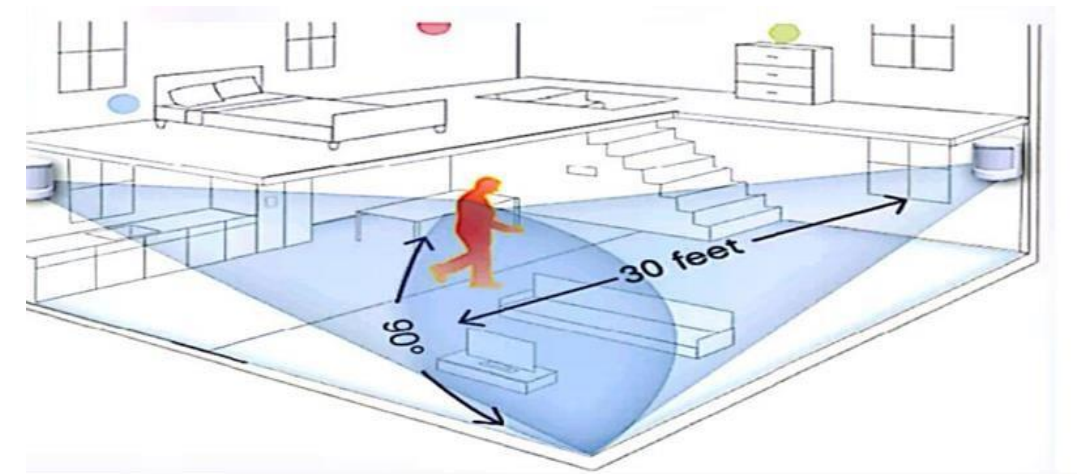

Figure 1: Active Infrared Sensor functional diagram [6].

2. Passive Infrared Sensor: a passive infrared sensor passively waits for an infrared event. When a heat source, like the human body, passes by, the sensor detects the moving presence.

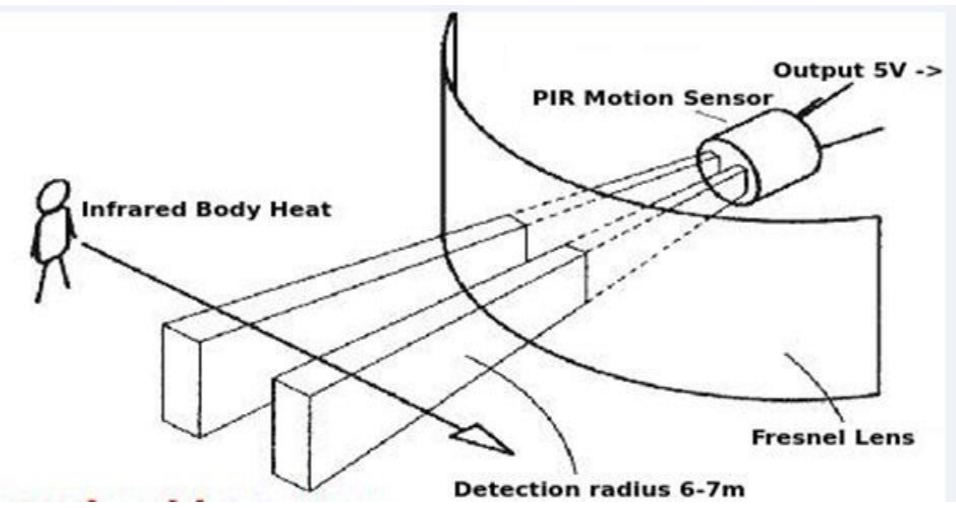

Figure 2: Passive Infrared Sensor functional diagram [7]. 


\section{IJARCCE}

3. Active Ultrasonic Sensors: Active ultrasonic sensors use high frequency sound waves that work like a boomerang these waves are sent out and then return to the sensor, reporting any suspicious interruptions in the sound wave [6].

4. Passive Ultrasonic Sensor: Instead of sending out sound waves these sensors wait passively for a sound event, the sound waves emitted by such an occurrence are what triggers a passive ultrasonic sensor.

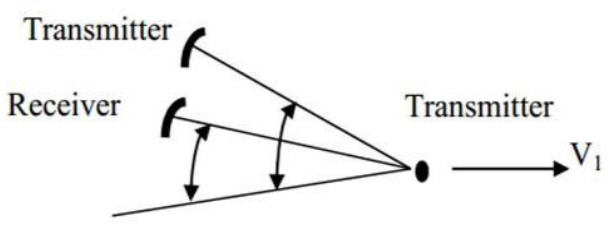

Figure 3: Ultrasonic Sensor Arrangement [8].

\subsection{Gas Sensors}

These detects gases which supports burning (fire), alcohol, toxic gases, and other gases. These sensing functions are based on designs that are derived from the two basic types of smoke detectors, namely:

a. The Photoelectric Smoke Detector

b. The Ionization Chamber Smoke Detector (ICSD) [9]

The photoelectric smoke detector uses an optical beam to search for smoke. When smoke particles cloud the beam, a photoelectric cell senses the decrease in light intensity and triggers an alarm. This type of smoke detector reacts most quickly to smouldering fires that release relatively large amounts of smoke.

On the other hand, the ionization chamber smoke detector is quicker at sensing flaming fires that produce little smoke. It employs a radioactive material to ionize the air in a sensing chamber, the presence of smoke affects the flow of the ions between a pair of electrodes, which triggers the alarm [9] [10]. In a typical system, the radioactive material emits alpha particles that strip electrons from the air molecules, creating positive oxygen and nitrogen ions. The electrons attach themselves to other air molecules, forming negative oxygen and nitrogen ions. Two oppositely charged electrodes within the sensing chamber attract the positive and negative ions, setting up a small flow of current in the air space between the electrodes, but when the smoke particles enter the chamber, they attract some of the ions, disrupting the current flow. There is usually a similar chamber constantly compares the current flow in the sensing chamber to the flow in the reference chamber; if a significant difference develops, an alarm is triggered. Constructed so that no smoke particles can enter, so that the smoke detector constantly compares the current flow in the sensing chamber to the flow in the reference chamber; if a significant difference develops, an alarm is triggered. This is the most commonly used design for domestic smoke detection [9].

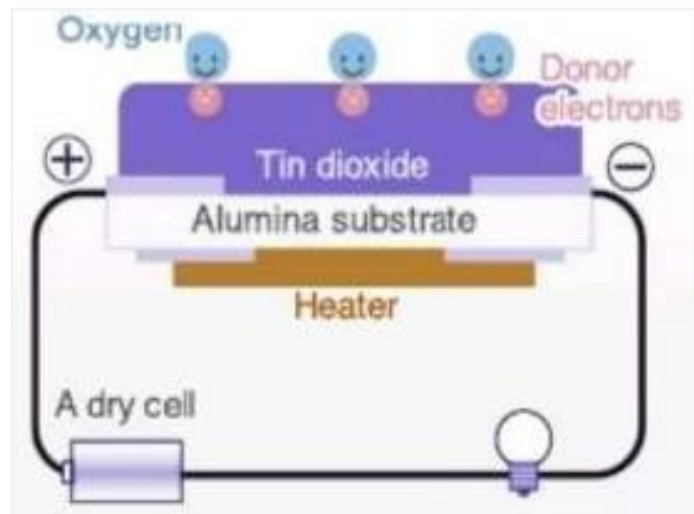

Figure 4: Internal Architecture of the MQ Gas Sensor series based on the ICSD [11].

\subsection{GSM/GPRS Module}

A GSM module is used to establish connection between a computer and a GSM/GPRS system. Global System for Mobile communication (GSM) is an architecture used for mobile communication in most of the countries. Global Packet Radio Service (GPRS) is an extension of GSM that enables higher data transmission rate [12]. GSM/GPRS module consists of a GSM/GPRS modem assembled together with power supply circuit and communication interfaces (like RS-232, USB, etc.). The soul of the module is the MODEM and it is wireless by design. Wireless MODEMs like other MODEM devices use serial communication to interface with computers and need compatible AT (attention) 
commands for communication with the microcontroller system. It requires a SIM (Subscriber Identity Module) card just like phones to activate communication with the network. A GSM MODEM can perform the following operations [12]:

1. Receive, send or delete SMS messages in a SIM.

2. Read, add, search phonebook entries of the SIM.

3. Make, Receive, or reject a voice call.

The MODEM needs AT (attention) commands, for interacting with a processor or controller.

\section{METHODOLOGY}

\section{a. HARDWARE UNITS AND INTERFACING}

The hardware part of this work, was divided into the following modules

- GSM sub-system prototype.

- Motion sensor sub-system prototype.

- Gas sensors sub-system prototype.

- $\quad$ Temperature sensor sub-system prototype.

This gave rise to the block diagram of the system being developed as;

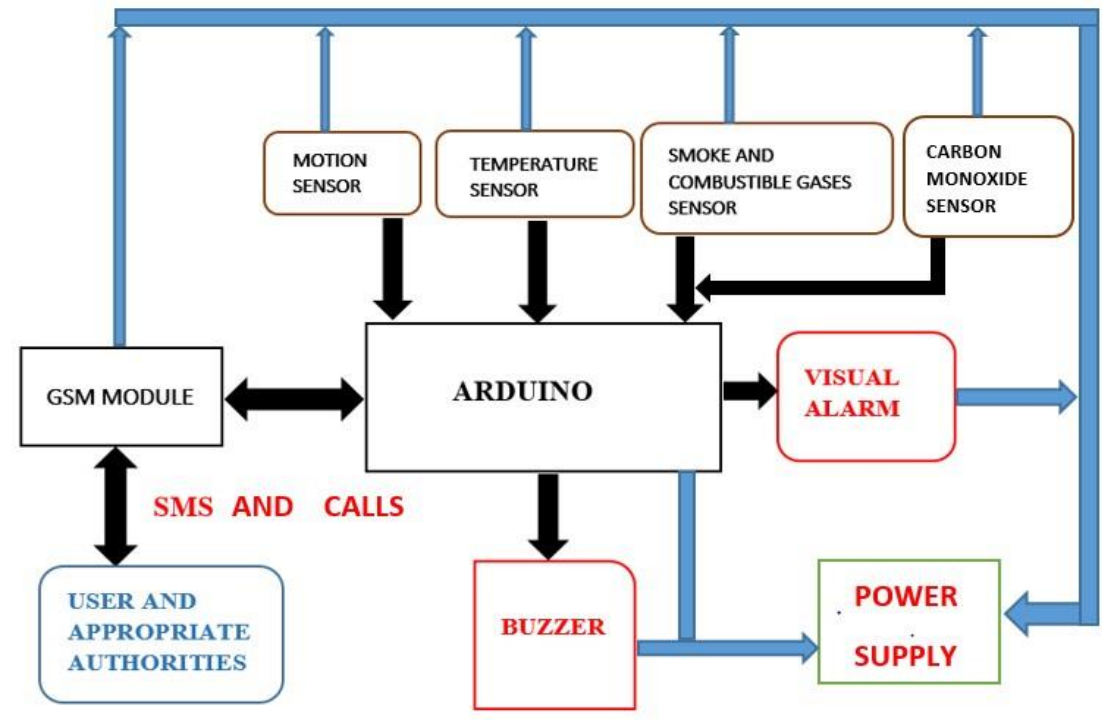

Figure 5: Block Diagram Design of the proposed system

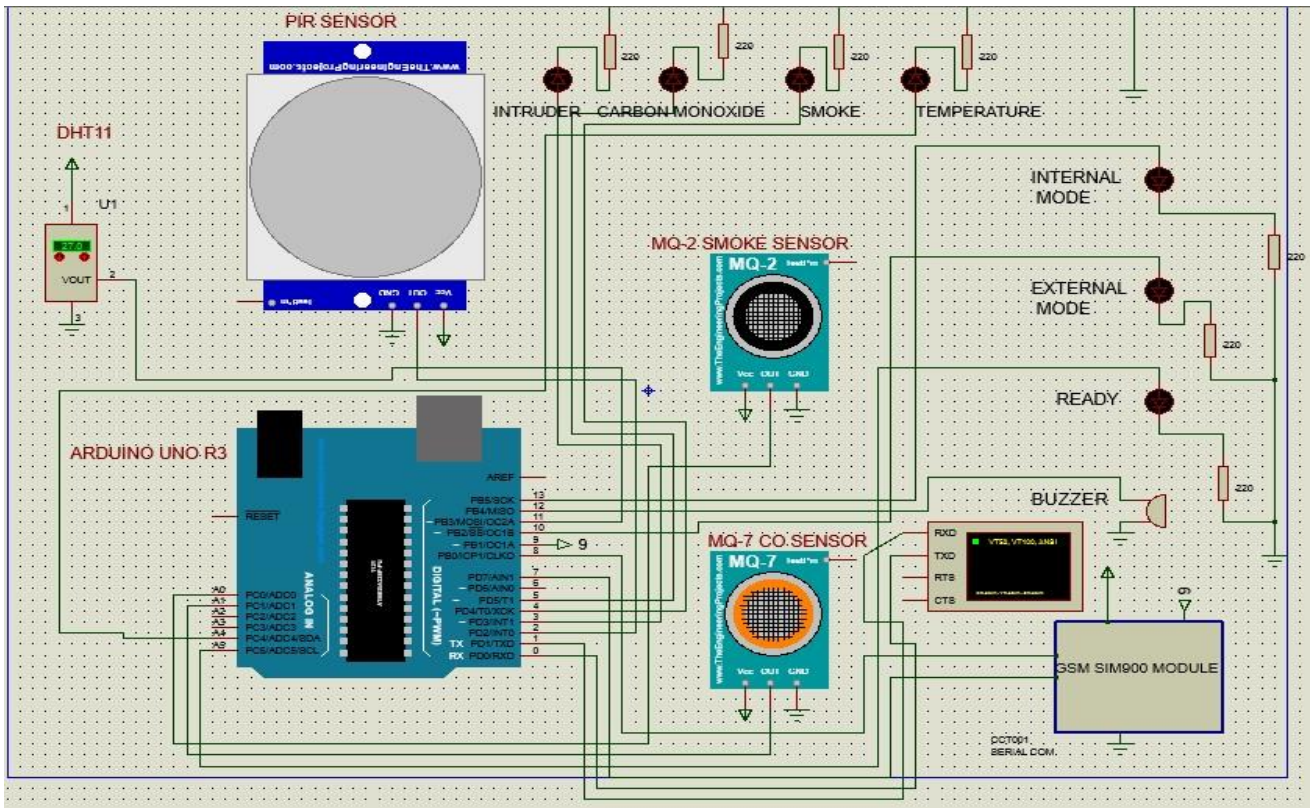

Figure 6: System Circuit Diagram Designed in Proteus 


\subsection{System Hardware Design}

The proposed GSM Based Smart Security System Using Arduino is a phone call switched, multi sensor-based system, comprising of a temperature sensor (LM35), a smoke/combustible gas sensor (MQ2), carbon monoxide sensor (MQ7), and an intrusion sensor (PIR) sections. These sensor sections work independently of themselves. The LM35 temperature sensor takes note of every abnormal rise in temperature above $45^{\circ} \mathrm{c}$, the MQ2 and MQ7 takes note of any dangerous concentration level of combustible gases/smokes and carbon monoxide respectively. The gas level threshold used for this work is when these gases make up $350 \mathrm{ppm}$. The PIR sensor depending on environmental conditions can have a sensitivity of about 20ft from its lenses. The AGSS 1 works in two modes of operation; an internal mode and an external operation mode. On power up, the system after initialization and (if) successful attempt of the GSM module to $\log$ on to a network provider's network, switches itself to a default internal mode. In the internal mode, the system assumes the owner is right at home or close by the said property, and as such the PIR sensor and the SMS capabilities of the system is disabled, leaving only the audio (buzzer) and visual (LEDs) alarms on the control panel, which is the central workhouse of the project, are triggered when any of the alarm conditions are met. The alarm conditions in this mode of operation are detected by the MQ2, MQ7 and LM35 sensors only. In the case of an unsuccessful attempt by the module to $\log$ on to the network during the given initialization period, the expected phone call, might come in, but won't be detected since it was not initialized properly.

The system periodically monitors the GSM module for any phone call, via software polling. In the case of a phone call, the Arduino checks the calling number, if the incoming caller ID corresponds to the phone number of the property owner which has been stored beforehand in the Arduino's ROM, then the system switches to the external operational mode, or the opposite operational mode to the one currently being executed. However, if the call is not from the prerecorded phone number the system ignores the call. The external operational mode, the PIR and text sending abilities of the GSM modules are activated, based on the assumption that the property owner is away. Thus, when alarming conditions are met, not only does the audio (buzzer) and visual (LEDs) alarms go off, but also the system sends an SMS with the sensed value of the threat to the owner, whilst in the case of intrusion a phone call is posted to the owner. During the period of alarm which lasts 15 seconds, no phone call is detected, in either mode. The designed system interestingly comprises no switch or physical button, switching of modes is entirely done by phone calls from the approved phone number. This is a security measure, to ensure that only the property owner has access to the system. Most hardware and functional features of the system are carefully thought out to reduce set-up and operational costs of the system. Thus, the system through its two-mode operation, offers flexibility of remote and immediate monitoring. Through its phone call switching mechanism, makes the system more secure and classy, using the SIM900 makes for a better power management and global adaptation.

\subsection{Arduino Unit}

For purpose of compatibility and cost, the Arduino Uno (the basic board) is preferred for most projects. The Arduino Uno is a microcontroller board based on the ATmega328. It has 14 digital input/output pins (of which 6 can be used as PWM outputs), 6 analog inputs, a $16 \mathrm{MHz}$ crystal oscillator, a USB connection, a power jack, an ICSP header, and a reset button. It contains everything needed to support the microcontroller; simply connect it to a computer with a USB cable or power it with an AC-to-DC adapter or battery to get started. , the Arduino Uno is shown below;

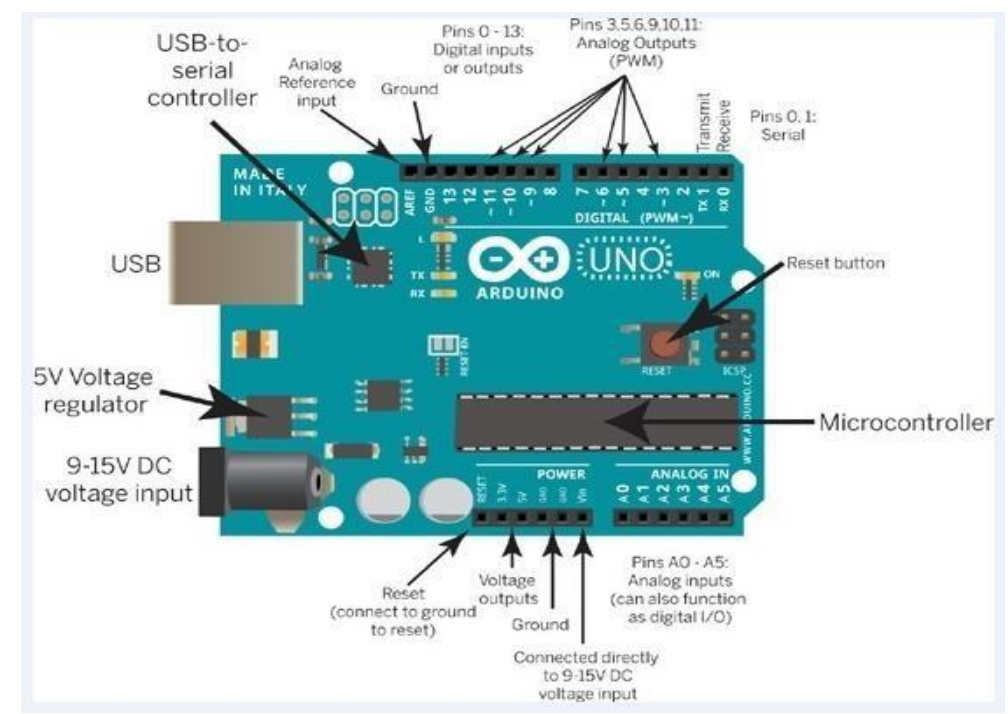

Figure 7: The Arduino Uno Board [8]. 


\title{
IJARCCE
}

\section{International Journal of Advanced Research in Computer and Communication Engineering}

\author{
Vol. 8, Issue 10, October 2019
}

\subsection{Motion Sensor Sub Circuit Design Analysis}

The PIR sensor proposed for this work is an Arduino designed generic PIR module, which is based on the Adafruit PIR sensor module shown below, the unit has the following key features [14];

- $\quad$ Single bit output

- $\quad$ Small size makes it easy to conceal

- $\quad$ Compatible with all Parallax microcontrollers

- $\quad 3.3 \mathrm{~V} \& 5 \mathrm{~V}$ operation with less than $100 \mathrm{uA}$ current draw

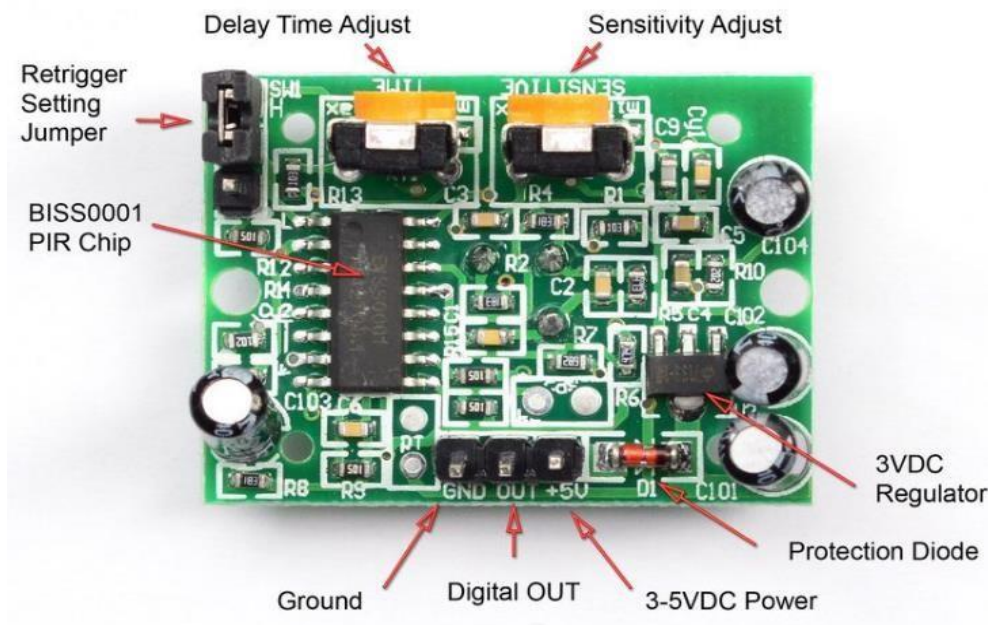

Figure 8: The PIR Module used in the Project [14].

The circuit of this unit of the system was developed and simulated first, it should be noted however that the PIR module used already have the protection diode, sensitivity and delay adjustment, the PIR chip etc, making for an easy usage. The PIR Sensor requires a 'warm-up' time in order to function properly. This could be anywhere from 10-60 seconds. This has been implemented in the software program.

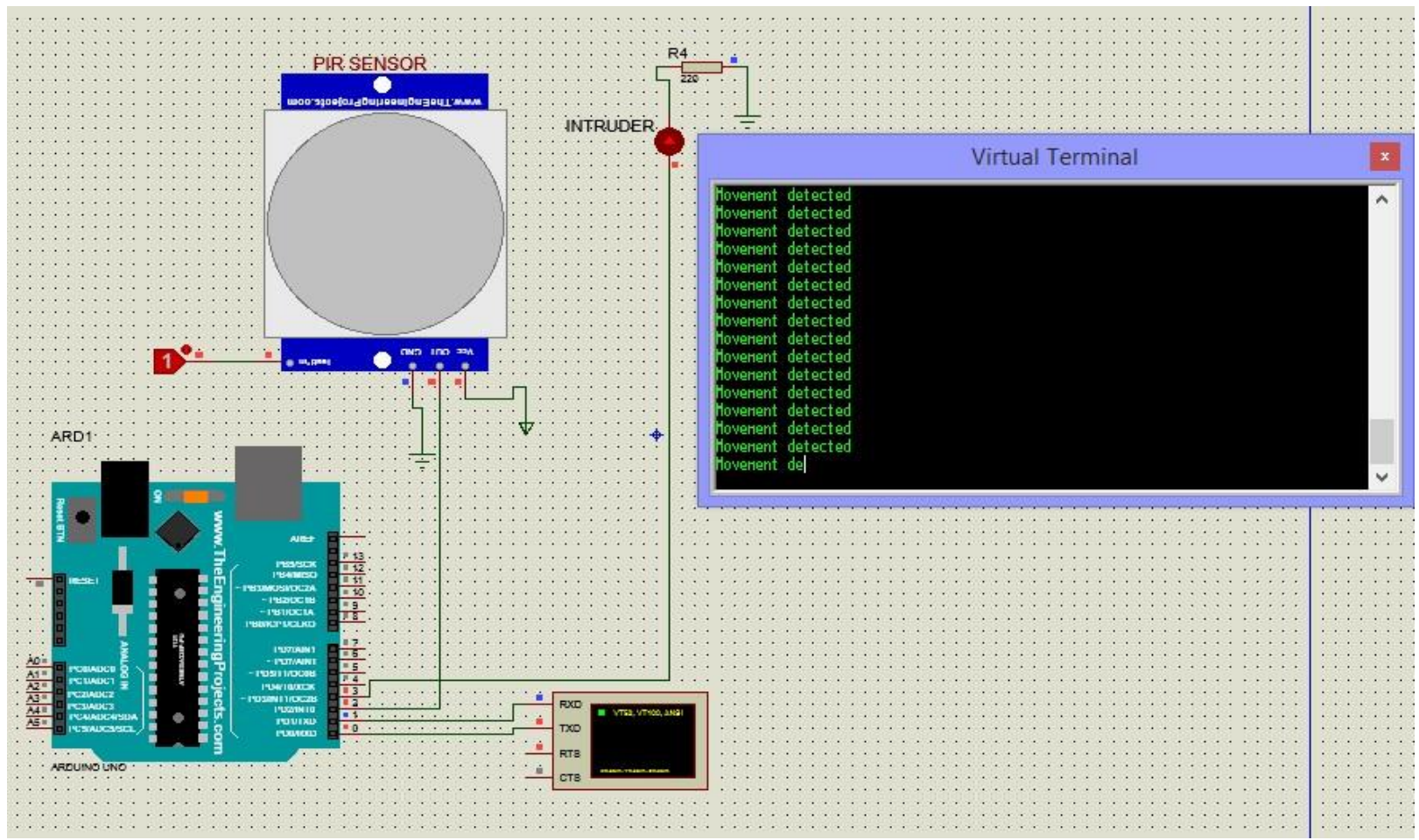

Figure 9: Motion Sensor Unit interface design, Simulation and results on Proteus.

3.4 Smoke and Combustible Gas Sensor Sub-Circuit Design Analysis

The interfacing of the MQ2 to the Arduino Uno is as shown 


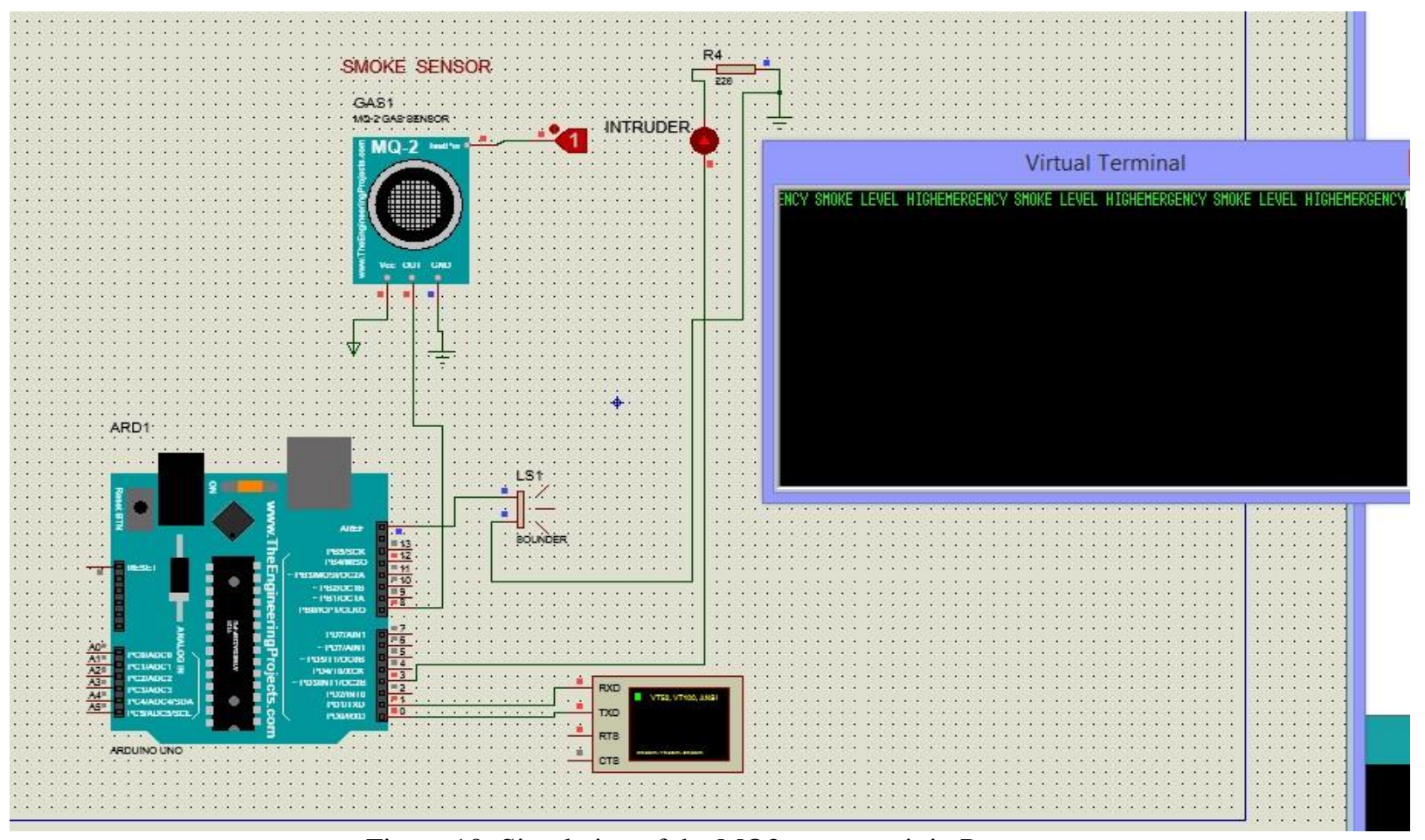

Figure 10: Simulation of the MQ2 sensor unit in Proteus

The design assigns an indicator LED to indicate that the sensor has sensed a value greater than the set threshold. The power requirement of the sensor can be from $2.5-5 \mathrm{~V}$, supply.

\subsection{Carbon Monoxide Gas Sensor Sub-Circuit Design Analysis}

The interfacing of this sensor to the Arduino is the same with the MQ2, the difference only lies in the physical feature and the detective gases. This unit is identical to the smoke sensing Unit above.

The resistor used in interfacing the LED is explained in section 3.7.

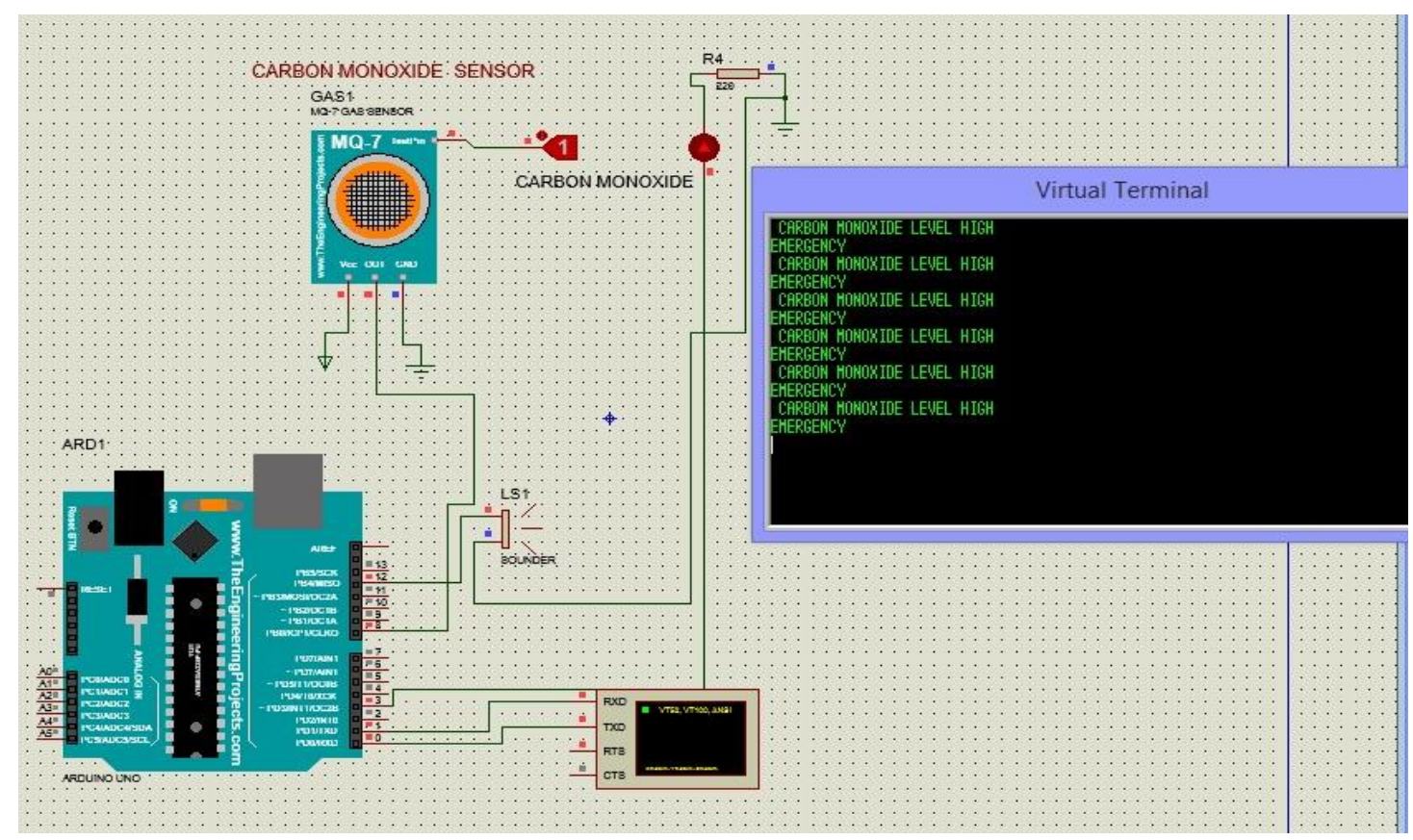

Figure 11: carbon monoxide sub circuit interfacing design and Simulation in Proteus.

\subsection{Temperature Sensor Unit Design Analysis}

The DHT11 digital temperature and humidity sensor is a composite Sensor which contains a calibrated digital signal output of temperature and humidity. The sensor is an Application of a dedicated digital modules collection technology 


\section{IJARCCE}

and the temperature and humidity sensing technology, to ensure that the product has high reliability and excellent longterm stability [15]. The sensor includes a resistive sense of wet components and an NTC temperature measurement device, and connected with a high-performance 8-bit microcontroller [15].
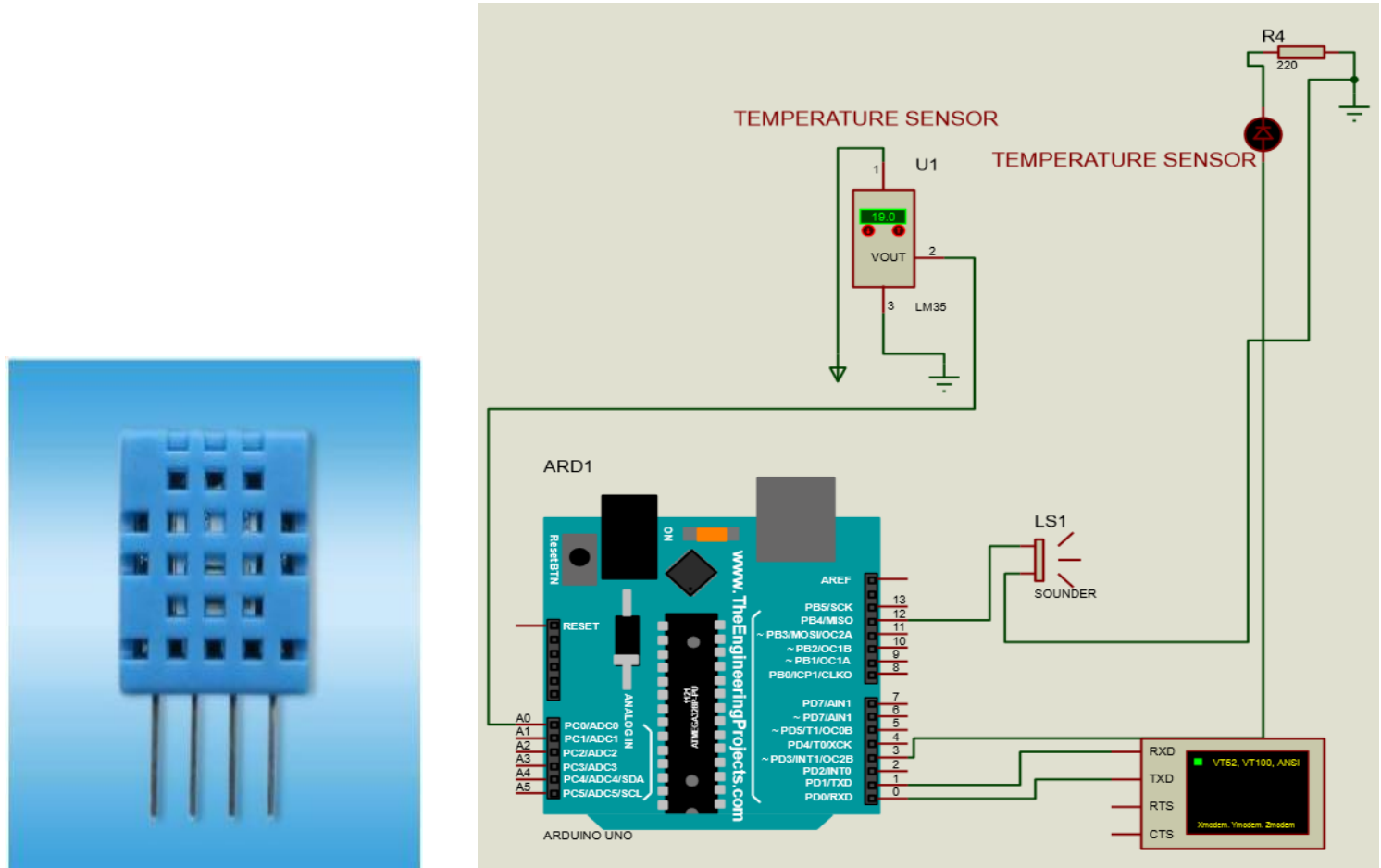

Figure 12: DHT-11 Temperature Sensor [15] and Temperature sensor sub unit designed in proteus.

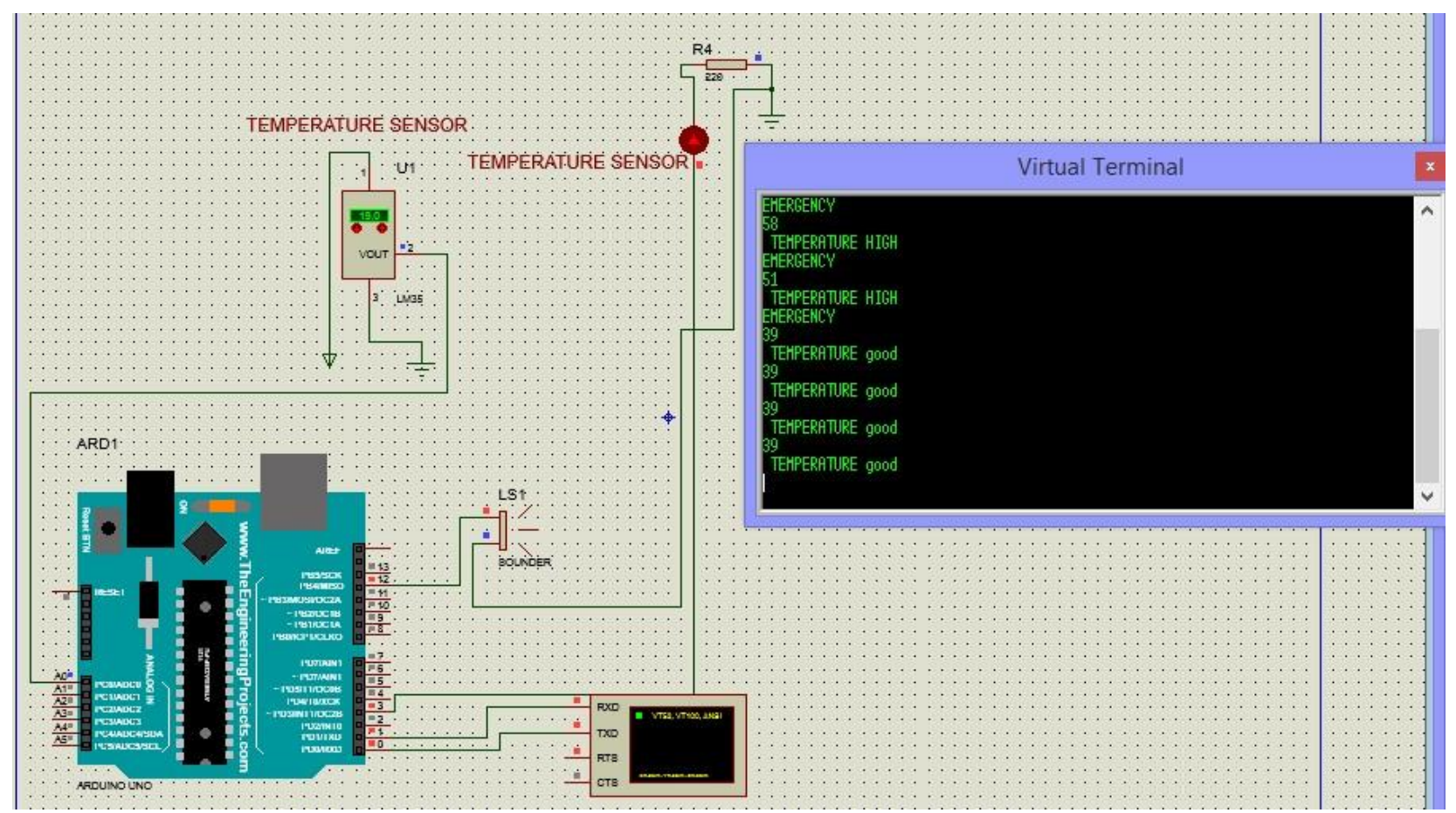

Figure 13: Simulation Results of the Unit in Proteus.

\subsection{Alarming Unit Sub Circuit Design and Analysis}

The alarm time is scheduled for 10 seconds and is set in software. 


\section{IJARCCE}

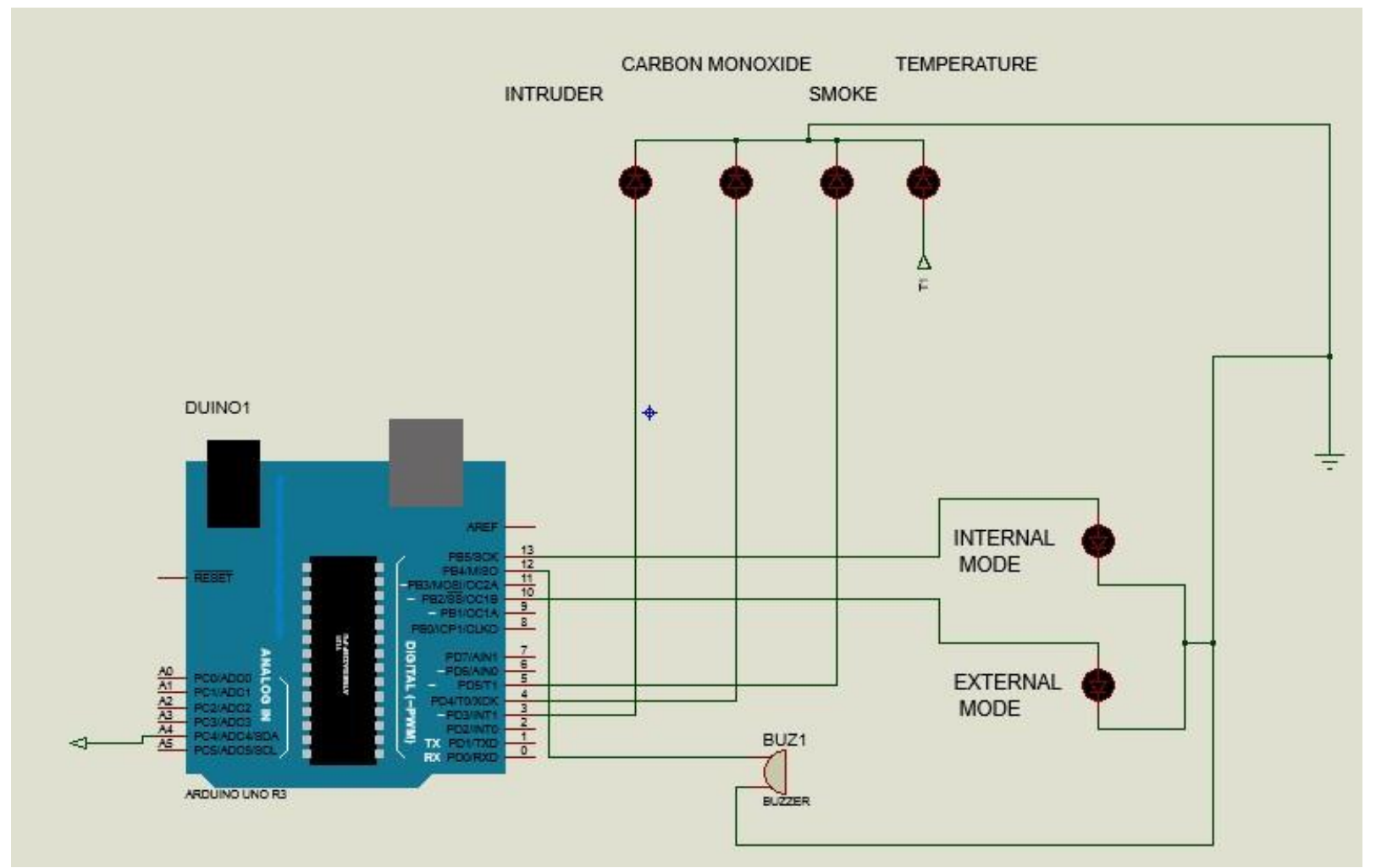

Figure 14: Alarming Sub-Circuit

After carefully considering the fact that the $5 \mathrm{v}$ from the Arduino Uno is much greater than the required and hence would damage the LEDs if not protected. This value is arrived at after applying the below formula (gotten from Kirchhoff's voltage law) for determining the series resistor that will divide the voltage

$$
\mathrm{R}=\frac{\text { Supply LED-LED voltage }}{\text { LED current }}
$$

The supply LED is the voltage, $5 \mathrm{v}$, supplied from the Arduino, the LED voltage is gotten from datasheet as $1.7 \mathrm{v}$ (red LED), and the current I wished to operate the LED at was $15 \mathrm{~mA}, 5 \mathrm{~mA}$ less than the maximum current of $20-25 \mathrm{~mA}$ specified for LED so as to prevent damage and at the same time getting a good brightness.

$$
R=\frac{5-1.7}{0.015}=220_{\Omega}
$$

The buzzer on the other hand is directly interfaced to the Arduino as it can handle the voltages of $5 \mathrm{~V}$ well as specified from the datasheet.

\subsection{Power supply Unit}

The Arduino requires a supply of 5-30V, while the SIM 900 module required from specifications 5V,1A for the nominal supply periods, thus a switch mode power supply of $9 \mathrm{~V}, 1 \mathrm{~A}$ was chosen for the work. The SIM 900 however has a considerably large current requirement during spike conditions, possibly reaching a high of $2 \mathrm{~A}$ (according to the datasheet), but this rarely happens, and even when it occurs, from tests carried out the 1A supply is more than capable of handling this.

\section{Software Design}

The design of the program to run the whole setup was hinged on the system algorithm as shown. This is the general system flowchart, which can be adopted on other hardware platforms. 
Vol. 8, Issue 10, October 2019

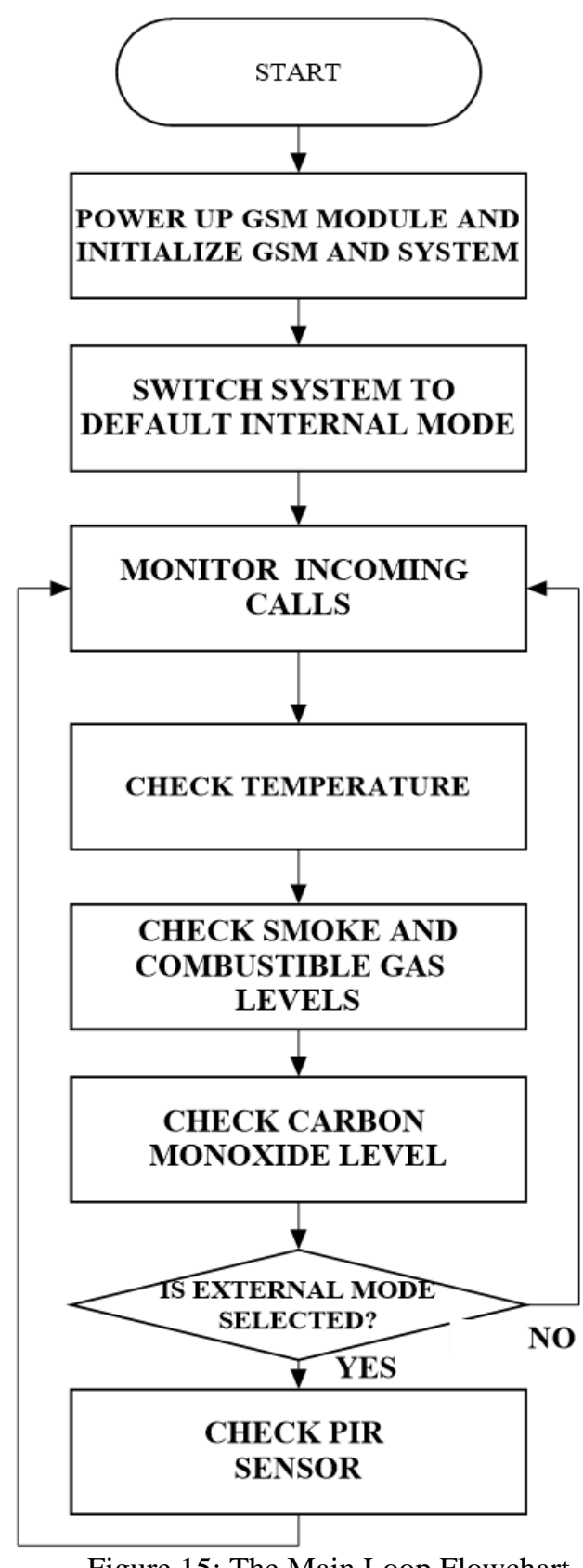

Figure 15: The Main Loop Flowchart.

\section{CONCLUSION}

Security as a core and vital part of human existence is crucial in promoting a healthy and sustainable environment that makes for peaceful and harmonious existence amongst the individuals and His society while also ensuring seamless interactions in a given habitat especially as it concerns lives and properties. The problems encountered by the traditional and old fashioned ways of security (like use of dogs, etc.) were corrected or remedied by the alarm systems. The alarm systems of old could not keep up with the dynamic changes from world traditional setting to a 'smart' setting. The older smart security systems only incorporated microcontrollers to replace electro-magnetic relays for control. The modern world however demands a smarter and more secure system of security that can alert the owner wherever he is, as well as the relevant authorities, to take immediate actions, and to be controlled and accessed only by the owner. It also allows the user access to control the system irrespective of locational or geographic barriers. This proposed system incorporates other forms of potential security threats into a single system thereby increasing effectiveness, flexibility, optimum performance and enhanced cost reduction while also increasing the scope of the coverage of security threats monitored and controlled. 


\section{REFERENCES}

[1]. Livio, "the English dictionary," http://wiktionary.org.

[2]. U.Mhen, "security systems," 23 MAY 2017. [Online]. www.wikipedia.com/security-system [Accessed 25 MAY 2017].

[3]. Arduino FAQ - With David Cuartielles". Malmö University "Arduino," 17 MAY 2017. [Online]. Available: www.wikipedia.com/arduino. [Accessed 21 O9 2017].

[4]. B. Evans, Begining Arduino Programming, Apress.

[5]. J. BOXALL, ARDUINO WORKSHOP: A Hands-On Introduction with 65 Projects, SAN FRANSISCO: NO STARCH PRESS, 2013 , p. 392.

[6]. C. Giaimo, "simplisafe," 19 June 2013. [Online]. Available: www.simplisafe.com/blog/motion-detectorguide. [Accessed 21 September 2017].

[7]. "DIYHACKING," JUNE 2014. [Online]. Available: www.diyhacking.com/pir-motion-sensor-automate-home/. [Accessed 27 may 2017].

[8]. u. sources images, "unknown," internet, google images, unknown.

[9]. N. R. NGOCHI, "SMOKE ALARM," UNIVERSITY OF NAIROBI, COLLEGE OF ENGINEEERING AND ARCHITECTURE, NAIROBI.

[10]. Madehow, "www.madehow.com," [Online]. Available: www.madehow.com/Volume-2/SmokeDetector.html. [Accessed 23 MAY 2017].

[11]. www.figaro.co.jp, "FIGARO Engineering Inc.," [Online]. Available: www.figaro.co.jp/en/technicalinfo/principle/mos-type.html. [Accessed 27 may 2017].

[12]. N. Agnihotri, "ENGINEERS GARAGE," 22 December 2010. [Online]. Available: www.engineersgarage.com/articles/gsm-gprs-modules. [Accessed 27 May 2017].

[13]. A. E. A. Radionics, Arduino-Uno (Datasheet).

[14]. Parallax (Datashheet), PIR Sensor (\#555-28027), PARALLAX INC, 2007.

[15]. Aosong Electronics Ltd., DATASHEET, Guangzhou, china: http://www.aosong.com. 